\title{
Optical Identification Strategies for the Molonglo Cluster Survey
}

\author{
A. J. Haigh, J. G. Robertson and R. W. Hunstead \\ School of Physics, University of Sydney, NSW 2006, Australia \\ ahaigh@physics.usyd.edu.au \\ Received 1996 October 26, accepted 1997 August 7
}

\begin{abstract}
We describe a newly refined procedure for making optical identifications of radio sources in Abell cluster fields observed with the Molonglo Observatory Synthesis Telescope (MOST). The method is based on past experience but uses a range of new tools to improve the reliability and production rate of identification lists. The COSMOS/UKST Southern Sky Object Catalogue was used to make preliminary identifications which were then inspected visually with the assistance of computer generated overlays of the MOST images and the Digitized Sky Survey (DSS). The overlaid images were essential for securing identifications for the extended sources prevalent among nearby clusters.

We find $21 \pm 1 \cdot 5 \%$ of the radio sources are identified with galaxies and $4 \cdot 6 \pm 0 \cdot 7 \%$ with QSO candidates in a sample of 927 radio sources in 27 cluster fields. We make a preliminary attempt to separate cluster radio galaxies from interlopers on the basis of absolute magnitudes. A strong concentration of radio galaxies was found at projected distances less than $100 \mathrm{kpc}$ from the cluster centres and a weaker concentration for projected distances of $100-500 \mathrm{kpc}$.
\end{abstract}

Keywords: galaxies: clusters, general — methods: data analysis

\section{Introduction}

Despite steady progress in the understanding of the radio emission from clusters of galaxies (e.g. Mills \& Hoskins 1977; Owen et al. 1982; Fanti et al. 1983; Reynolds 1986; Andernach et al. 1988; Unewisse 1993; Slee, Roy \& Savage 1994; Ledlow \& Owen $1995 a, 1995 b, 1996)$, it is still not clear what effect, if any, the cluster environment has on the likelihood of a galaxy becoming a strong radio emitter. In recent years one of the major observing programs on the Molonglo Observatory Synthesis Telescope (MOST; see Robertson 1991, and references therein, for a description) has been a survey of 261 southern clusters from the catalogue of Abell, Corwin \& Olowin (1989; ACO), with the aim of extending our current knowledge of the radio properties of clusters. The survey samples two overlapping subsets of the ACO catalogue. The first consists of all clusters with a distance class $\mathrm{D} \leq 4$, equivalent to redshifts $z \leq 0 \cdot 072$. The second consists of all clusters with a Molonglo Reference Catalogue source (MRC; Large et al. 1981) lying within a projected separation of $670 \mathrm{kpc}$ from the centre $\left(H_{0}=75 \mathrm{~km} \mathrm{~s}^{-1} \mathrm{Mpc}^{-1}\right)$, as listed by Robertson \& Roach (1990).

One advantage of the Molonglo Cluster Survey over previous radio surveys arises from the wide $70^{\prime} \times 70^{\prime} \operatorname{cosec}(\delta)$ field of view of the MOST, which covers an area out to the Abell radius $R_{A}(\mathrm{ACO})$ when centred on clusters at redshift $z \geq 0 \cdot 048$. The sensitivity of the MOST (the weakest catalogued sources being $\sim 5-10 \mathrm{mJy}$ ) is about the same as the most recent previous surveys mentioned above. In addition, the observing frequency of $843 \mathrm{MHz}$ is suitable for detection of steep spectrum sources which are most often found in clusters, and are less readily detected at higher frequencies. Another advantage is the excellent ability of the MOST to image the extended, diffuse structures (such as the rare halo and relic sources) peculiar to cluster fields.

In deep radio surveys there are far fewer sources optically identified with cluster members than there are faint, random background sources. For this reason, in some earlier surveys the likely number of cluster-related sources at a given radius from the cluster centre was estimated from the statistical excess over the background. Ideally, we would like to select only those sources associated with the clusters, in order to gain insight into the physics that governs the radio properties of cluster galaxies. Selection of cluster sources first requires reliable optical identifications. This paper presents an identification method developed and tested on a sample of 27 clusters from the MOST survey. Its major advantage is the use of relatively new tools, such as the COSMOS/UKST Southern Sky Object Catalogue and the Digitized Sky Survey, resulting in more efficient and reliable optical identifications than were possible in the past. 
Table 1. The sample of clusters studied in this paper

\begin{tabular}{|c|c|c|c|c|c|c|c|c|c|c|}
\hline Cluster & $\begin{array}{c}\mathrm{RA} \\
(\mathrm{J} 2000)\end{array}$ & $\begin{array}{c}\text { Dec } \\
(\mathrm{J} 2000)\end{array}$ & $\begin{array}{l}\text { Abell } \\
\text { type }\end{array}$ & $\begin{array}{l}\mathrm{B}-\mathrm{M} \\
\text { type }\end{array}$ & $\mathrm{R}$ & $\mathrm{D}$ & $m_{10}$ & $z$ & $\begin{array}{c}\mathrm{rms}(\text { full }) \\
(\mathrm{mJy} / \text { beam })\end{array}$ & $\begin{array}{l}\text { rms (inner) } \\
(\mathrm{mJy} / \text { beam) }\end{array}$ \\
\hline A2731 & $\begin{array}{lll}00 & 10 & 12\end{array}$ & -5659 & I & III & 0 & 3 & $15 \cdot 3$ & $0 \cdot 0312$ & $1 \cdot 28$ & $0 \cdot 99$ \\
\hline S52 & 002742 & -5411 & IR & II & 0 & 5 & $17 \cdot 2$ & $0 \cdot 104^{*}$ & $1 \cdot 13$ & $1 \cdot 10$ \\
\hline A2806 & $\begin{array}{lll}00 & 40 & 12\end{array}$ & -5609 & RI & I-II & 0 & 3 & $15 \cdot 3$ & $0 \cdot 0271$ & $1 \cdot 13$ & $0 \cdot 91$ \\
\hline A3125 & 032724 & -5330 & I & III & 0 & 4 & $15 \cdot 8$ & $0 \cdot 0593$ & $2 \cdot 13$ & $1 \cdot 66$ \\
\hline A3128 & $\begin{array}{lll}03 & 30 & 12\end{array}$ & -5233 & RI & I-II & 3 & 3 & $15 \cdot 3$ & $0 \cdot 0554$ & $1 \cdot 14$ & $1 \cdot 01$ \\
\hline A3144 & 033706 & -5501 & IR & I-II & 1 & 4 & $15 \cdot 8$ & $0 \cdot 0423$ & $1 \cdot 21$ & $0 \cdot 88$ \\
\hline A3164 & 034548 & -5702 & IR: & I-II & 0 & 4 & $15 \cdot 7$ & $0 \cdot 0611$ & $1 \cdot 21$ & $0 \cdot 95$ \\
\hline A3202 & $\begin{array}{lll}04 & 00 & 12\end{array}$ & -5339 & I & II & 1 & 4 & $15 \cdot 8$ & $0 \cdot 0338$ & $1 \cdot 29$ & $0 \cdot 86$ \\
\hline $\mathrm{S} 463$ & 042912 & -5349 & $\mathrm{R}:$ & I-II & 0 & 3 & $15 \cdot 3$ & $0 \cdot 0394$ & $2 \cdot 13$ & $2 \cdot 69$ \\
\hline $\mathrm{S} 522$ & $0518 \quad 24$ & -5613 & RI & I-II & 0 & 5 & $16 \cdot 9$ & $0 \cdot 092^{*}$ & $1 \cdot 50$ & $1 \cdot 82$ \\
\hline A3391 & $\begin{array}{lll}06 & 26 & 18\end{array}$ & -5340 & $\mathrm{R}$ & I & 0 & 4 & $16 \cdot 1$ & $0 \cdot 0531$ & $1 \cdot 78$ & $3 \cdot 47$ \\
\hline $\mathrm{A} 3395^{1}$ & 062730 & -5423 & $\mathrm{R}$ & II & 1 & 4 & $15 \cdot 9$ & $0 \cdot 0498$ & $2 \cdot 84$ & $2 \cdot 49$ \\
\hline A3651 & 195212 & -5505 & RI: & II & 1 & 3 & $15 \cdot 4$ & $0 \cdot 0588$ & $1 \cdot 72$ & $1 \cdot 52$ \\
\hline S839 & 200118 & -5255 & IR & I-II: & 0 & 3 & $15 \cdot 4$ & $0 \cdot 0526$ & $1 \cdot 58$ & $1 \cdot 93$ \\
\hline S840 & 200324 & -5557 & RI: & I-II: & 0 & 1 & $13 \cdot 9$ & $0 \cdot 0152$ & $0 \cdot 76$ & $1 \cdot 25^{*}$ \\
\hline S854 & 201124 & -5644 & IR & II & 0 & 4 & $15 \cdot 7$ & 0.0536 & $1 \cdot 54$ & $1 \cdot 61$ \\
\hline A3667 & 201230 & -5648 & IR: & I-II & 2 & 3 & $15 \cdot 4$ & $0 \cdot 0552^{2}$ & $1 \cdot 18$ & $1 \cdot 39^{*}$ \\
\hline A3716 & 205130 & -5242 & IR & I-II: & 1 & 3 & $15 \cdot 0$ & $0 \cdot 0456$ & $1 \cdot 79$ & $1 \cdot 50$ \\
\hline A3785 & 213430 & -5337 & I & II & 0 & 4 & $16 \cdot 2$ & $0 \cdot 0775$ & $1 \cdot 44$ & $1 \cdot 66$ \\
\hline A3806 & 214636 & -5717 & $\mathrm{R}$ & II & 2 & 4 & $16 \cdot 2$ & $0 \cdot 0747$ & $1 \cdot 07$ & $0 \cdot 92$ \\
\hline A3816 & 215024 & -5518 & I & I-II & 0 & 3 & $15 \cdot 3$ & $0 \cdot 0352$ & $1 \cdot 44$ & $1 \cdot 29$ \\
\hline A3826 & 215954 & -5609 & I & II & 1 & 3 & $15 \cdot 1$ & $0 \cdot 0754$ & $1 \cdot 35$ & $1 \cdot 18$ \\
\hline A3851 & $22 \quad 1642$ & -5235 & I & I-II & 0 & 4 & $16 \cdot 0$ & $0 \cdot 0529$ & $1 \cdot 21$ & $1 \cdot 13$ \\
\hline A3869 & 222124 & -5507 & I & II: & 0 & 3 & $15 \cdot 3$ & $0 \cdot 0396$ & $1 \cdot 22$ & $0 \cdot 95$ \\
\hline A3886 & 223142 & -5444 & I & III & 0 & 4 & $17 \cdot 3$ & $0 \cdot 0750$ & $1 \cdot 22$ & $1 \cdot 06$ \\
\hline S1039 & 223454 & -5227 & I & I-II: & 0 & 4 & $16 \cdot 4$ & $0 \cdot 0554$ & $1 \cdot 44$ & $1 \cdot 45$ \\
\hline A3911 & 224606 & -5243 & RI: & II-III & 1 & 5 & $17 \cdot 1$ & $0 \cdot 100^{*}$ & $1 \cdot 09$ & $0 \cdot 94$ \\
\hline S1115 & 232200 & -5446 & $\mathrm{R}$ & III & 0 & 6 & $17 \cdot 6$ & $0 \cdot 123^{*}$ & $1 \cdot 78$ & $1 \cdot 93$ \\
\hline
\end{tabular}

${ }^{1}$ Not in sample (see Section 2.1). ${ }^{2}$ From Sodré et al. (1992).

Section 2 describes the procedure for cataloguing the radio sources in the MOST images. Section 3 presents an automated method for making optical identifications on the basis of positional coincidence and for determining their reliability. Section 4 discusses radio-optical overlays as the only viable method for identifying extended, well resolved sources which make up a small but important minority of our source lists. In Section 5 we present the results of the identification procedure, and compare them with earlier results from Unewisse (1993). Section 6 outlines a first attempt to separate the cluster and non-cluster radio galaxies, and Section 7 states our conclusions.

Throughout this paper a Hubble constant of $H_{0}=75 \mathrm{~km} \mathrm{~s}^{-1} \mathrm{Mpc}^{-1}$ is assumed, giving an Abell radius of $R_{A}=2 \mathrm{Mpc}$.

\section{Radio Source Catalogue}

\subsection{The Cluster Sample}

The sample to be studied was chosen as a complete, representative subset of the overall survey. This was achieved by selecting the 28 clusters within a $5^{\circ}$ declination strip centred on $-55^{\circ}$. Details of the clusters are listed in Table 1. All data are from ACO unless otherwise noted. The columns are as follows:

Column 1: The cluster name.

Columns 2 \& 3: Coordinates of the cluster centre.
Column 4: The Abell type; a colon indicates a mean type or uncertain type estimate.

Column 5: The Bautz-Morgan type; a colon means uncertain type.

Column 6: The Abell richness class, R.

Column 7: The Abell distance class, D.

Column 8: The magnitude of the tenth brightest galaxy in the cluster, $m_{10}$

Column 9: The best available redshift. If no redshift has been measured, the one estimated from $m_{10}$ (by ACO) is shown, indicated by an asterisk $\left(^{*}\right)$.

Columns 10 and 11: The pixel value rms in units of $\mathrm{mJy} /$ beam of the entire MOST radio image, and that of the central $23^{\prime}$, using iterative $2 \sigma$ clipping on a histogram of image pixel values. When the inner field value was unavailable, the rms of a source-free region near the field centre was obtained, using the AIPS task IMEAN, and used instead. These values are indicated with an asterisk $\left(^{*}\right)$.

The image quality for one cluster (A3395) was badly affected by sidelobes from a strong source in A3391 and was not used in the current analysis. Therefore, the sample on which the identification process was carried out contains 27 cluster fields.

\subsection{Source Fitting}

The new source-fitting program VSAD (W. Cotton 1995, private communication), developed for the 
NRAO VLA Sky Survey (NVSS), was incorporated into AIPS and used to compile preliminary source lists. VSAD is an enhancement of the standard AIPS task SAD; it fits elliptical Gaussians, as described by Condon (1996), and subtracts the fitted components from the image to form a residual image. It performed well on the majority of MOST images. In particular, for many extended and closely blended sources there was far less evidence of fitting errors than with SAD. All residual images were carefully inspected and in cases where fitting errors were evident, peak positions and flux densities were found using the AIPS verb MAXFIT and integrated flux densities were found using TVSTAT, which integrates the total flux in a polygonal area defined by the user. Whenever the peak flux density values given by VSAD and MAXFIT differed by more than $2 \%$ for strong (>50 mJy) unresolved sources, the VSAD value was replaced by the maXfit value. The same was done for integrated flux densities using tVSTAT. This ensured that for strong sources any uncertainty in these quantities from the fitting was smaller than the calibration uncertainty inherent in the MOST, which is typically about $5 \%$. The calibration error was established by comparing the strong sources in 12 pairs of repeat observations from the large overall survey dataset. A larger relative difference was allowed for weaker sources $(<20 \mathrm{mJy})$ where flux density uncertainties are becoming dominated by noise. Where the positions differed by more than about $20^{\prime \prime}$ the MAXFIT positions were preferred.

To ensure reliability of the source lists, a $5 \sigma$ cutoff was applied to the peak flux densities. The value of $\sigma$ was chosen conservatively. In most cases, we adopted the larger of the two estimates given in Table 1. In the extreme case of A3391, the smaller full-field estimate was used because the larger inner value was boosted by radial artifacts associated with the strong central source; visual inspection was used to check the reality of catalogued sources in this inner region. All images were similarly inspected to ensure that image artifacts or sidelobe confusion were not mistaken for sources. Where confusion was evident the flux density errors were increased substantially over those derived in the next section.

\subsection{Uncertainties}

The 12 repeat observations mentioned above were used as a check on the uncertainties given by VSAD. It was found that the position differences could be modelled by the combination in quadrature of a systematic (calibration) offset, due to residual pointing errors, and a random scatter. Figure 1 shows that the scatter in both $\alpha$ and $\delta$, after removing the systematic offsets, depends inversely on peak flux density up to some level, above which
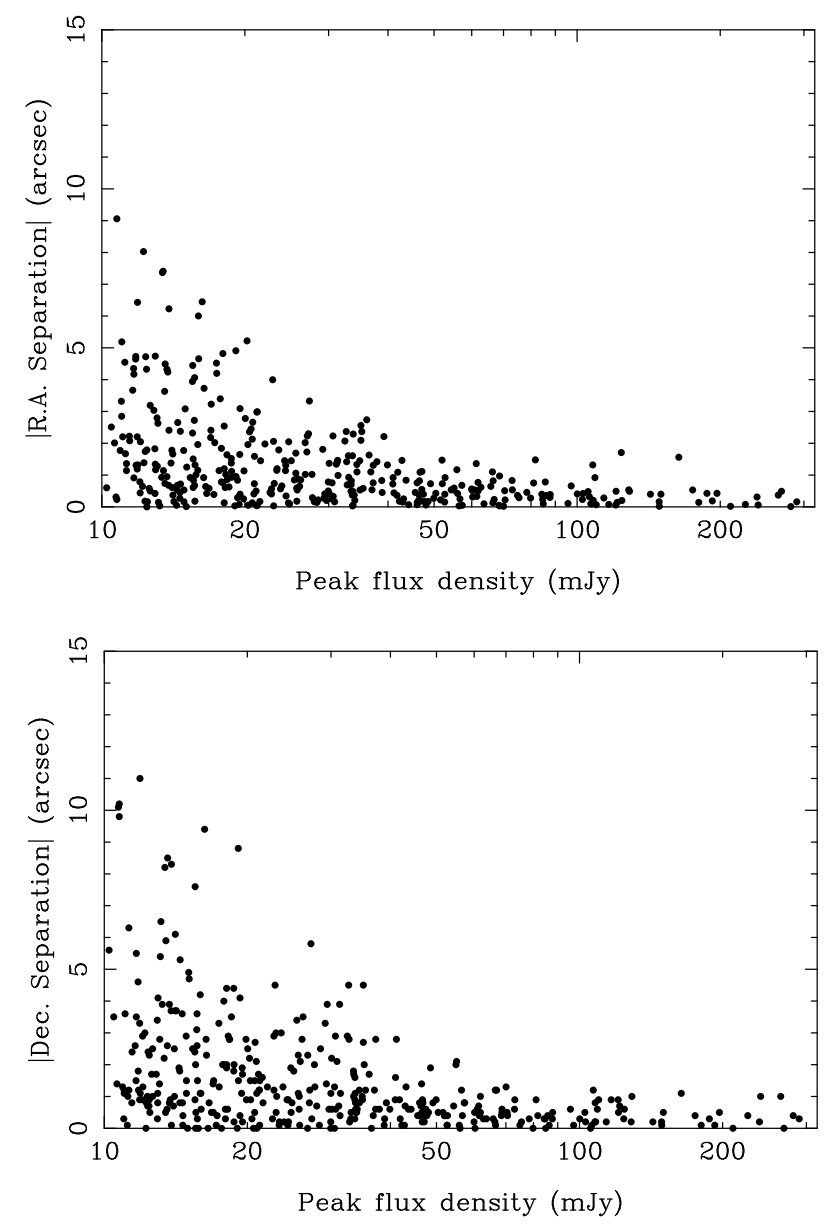

Figure 1-Differences in RA (top) and Dec (bottom) between pairs of observations of identical sources (after subtraction of systematic offsets and removal of obvious outliers), as a function of peak flux density. The variances in RA and Dec differences were fitted to equation (1) to define the noise component of position uncertainties.

a constant term dominates. The model can be described by the equation

$$
\sigma^{2}=A^{2} / S^{2}+B^{2}
$$

where $\sigma^{2}$ is the variance of the differences in RA or Dec for a pair of observations at a given peak flux density; $A$ represents the error due to the overall signal-to-noise, $B$ represents the calibration error, and $S$ is the peak flux density. In general there are approximately equal contributions to $A$ from thermal noise and confusion. However, there are some regions where sidelobe confusion (e.g. grating rings) dominates. As mentioned in Section $2 \cdot 2$, only those sources deemed to be reliable detections were included in the error analysis. While $A$ and $B$ would normally be obtained directly from a fit to equation (1), in the present case $B$ was set first by the rms scatter among different pairs of strong sources, and $A$ was then found from fitting. For RA differences we find $A=39 \operatorname{arcsec}$ mJy and $B=1.3 \operatorname{arcsec}$, and for Dec differences $A=49 \operatorname{cosec}(\delta) \operatorname{arcsec} \mathrm{mJy}$ and 
$B=1 \cdot 2 \operatorname{cosec}(\delta) \operatorname{arcsec}$. The $\operatorname{cosec}(\delta)$ factor comes from the shape of the synthesised beam, assuming that errors are a fixed fraction of the beamwidth. The position uncertainties $\sigma_{\alpha}$ and $\sigma_{\delta}$ are then given by $\sigma / \sqrt{2}$ for a source of peak flux density $S$. The formal position errors from VSAD were found to be similar to the flux density dependent component of errors determined empirically.

\section{Identifications with COSMOS}

The COSMOS/UKST Southern Sky Object Catalogue is a database containing parameters of optical objects, formed by scanning the blue IIIaJ plates from the UK Schmidt Southern Sky Survey; see Collins, Heydon-Dumbleton \& MacGillivray (1989), and references therein, for a description. The catalogue is available on-line at the Anglo-Australian Observatory $^{1}$ and a description of the software used to extract data and finding charts is given by Drinkwater, Barnes \& Ellison (1995). An improvement to the astrometric accuracy of the catalogue (by correcting an error in the coordinate system transformation) has been implemented in the on-line software following the discovery of systematic offsets by Unewisse, Hunstead \& Piestrzynski (1993).

The great advantage in using such a catalogue for identification work is that it is a fast process, and gives many useful parameters for the objects. However, it does contain errors, the most common being misclassification of a small fraction of galaxies as stars and vice versa, and the occasional omission of objects altogether. The software that produces the catalogue can also have trouble deblending partly merged images. For a discussion of these and other issues see Unewisse, Hunstead \& Piestrzynski (1993). By combining COSMOS identifications with inspection of the sky survey images, discussed in Section 4 , we can expect to obtain a more complete and reliable list of identifications than one made with COSMOS alone.

\subsection{Automated Identifications}

A preliminary list of optical identifications for all of the sources found in the MOST fields was compiled by extracting object lists and $2^{\prime} \times 2^{\prime}$ finding charts centred on each radio position from the COSMOS catalogue. The uncertainties derived from equation (1) were adopted for the radio positions. The uncertainty in the COSMOS positions is quoted at $\sim 1-2^{\prime \prime}$ (Unewisse et al. 1993) but has been improved somewhat by Drinkwater et al. (1995). A conservative value of $1^{\prime \prime} .5$ was adopted for both $\alpha$ and $\delta$. Each object within $30^{\prime \prime}$ of the radio position was assessed for plausibility as a possible identification according to the following criteria. Following Allington-Smith

\footnotetext{
1 http://www.aao.gov.au/local/www/surveys/cosmos/
}

et al. (1982), we calculated the radio-optical position difference $R$, normalised by the combined errors, as follows:

$$
R=\left[\frac{(\Delta \alpha)^{2}}{\sigma_{\alpha o}^{2}+\sigma_{\alpha r}^{2}}+\frac{(\Delta \delta)^{2}}{\sigma_{\delta o}^{2}+\sigma_{\delta r}^{2}}\right]^{\frac{1}{2}}
$$

Here $\Delta \alpha$ and $\Delta \delta$ are the separations in RA and Dec respectively, $\sigma_{\alpha o}$ and $\sigma_{\delta o}$ are the uncertainties in the optical positions ( $\left.1^{\prime \prime} .5\right)$, and $\sigma_{\alpha r}$ and $\sigma_{\delta r}$ are the uncertainties in the radio positions (equation $1)$. For galaxies, an identification was deemed to be genuine if $R \leq 3$. For unresolved and slightly resolved sources, to which this method can validly be applied, Allington-Smith et al. (1982) showed that this gives a formal completeness of $99 \%$. For stellar objects and so-called 'faint' objects (objects too faint for reliable classification), the identification was deemed to be genuine if $R \leq 1$. This gives a lower completeness for these objects, but the high surface density of stellar objects would result in an unacceptably high number of chance coincidences if the $R \leq 3$ criterion was applied, as discussed in Section $3 \cdot 2$. Although the set of galaxy identifications of unresolved sources obtained purely from the method described here is highly complete, it is not fully reliable. Section $3 \cdot 2$ describes measures taken to ensure reliability of the identification list.

\subsection{Chance Coincidences}

To assist in deciding which of the preliminary identifications to accept, estimates were made of the probability that any coincidence of radio and optical objects is real or due to chance. The online COSMOS software at AAO was used to find the closest optical counterparts to all the fitted radio positions in the subsample of cluster fields being studied. The same was done for positions offset from the true positions by $2-10^{\prime}$, with the data from 40 different offset vectors combined to improve the statistics. Figure 2 shows the normalised detection frequency of stellar objects and galaxies as a function of separation from the true positions, i.e. the probability that an association has occurred by chance. The main conclusions from Figure 2 and additional plots (not shown) were:

(1) The probability of finding a genuine stellar identification is very small at radio-optical separations greater than $5^{\prime \prime}$ (Figure 2a). The same result is found for 'faint' objects.

(2) Galaxies have a much higher probability of being genuine identifications than stellar objects (compare Figures $2 \mathrm{a}$ and $2 \mathrm{~b}$ ). This is consistent with past work which has shown that radio galaxies are more numerous than quasars in a low frequency survey. No radio stars are expected to be detected to our sensitivity limit. 

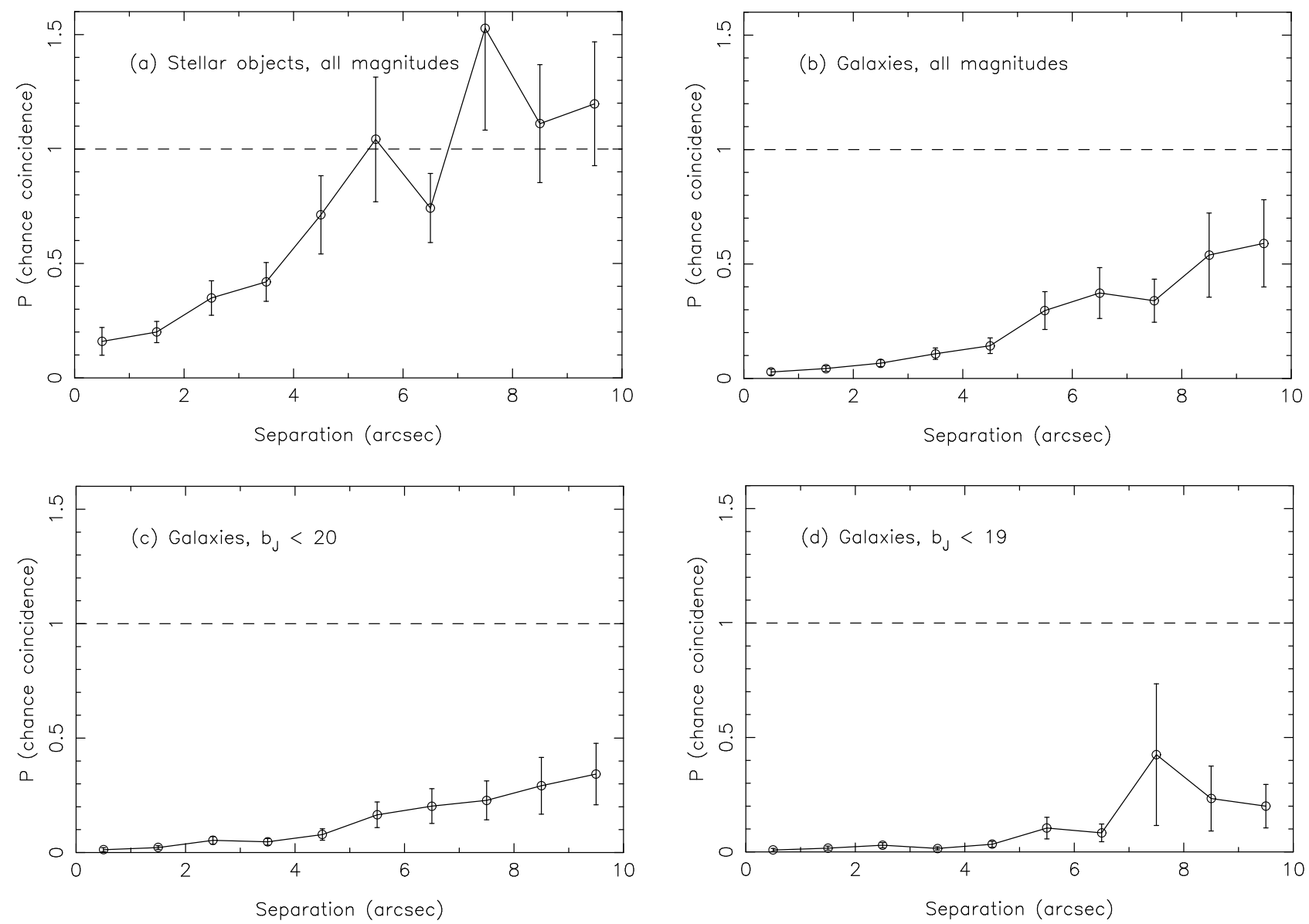

Figure 2-Probability that the closest object to a radio position is a chance association, as a function of radio-optical separation: (a) for stellar objects and (b), (c) and (d) for galaxy samples with different $b_{J}$ magnitude limits.

(3) At a given radius vector offset, the likelihood that a galaxy association is real increases as brighter magnitude cutoffs are applied.

(4) Because of the broad distribution of quasar optical luminosities, magnitude is generally not a good indicator of the reliability of a candidate stellar identification.

Although these are useful conclusions, they are based on the average density of objects in all the fields when in fact there is significant variation from field to field. To establish a definite reliability limit, the density of galaxies, stars and faint objects was calculated in each individual field. This better accounts for the variation of galaxy density with magnitude in clusters of different richness and redshift, and the variation of stellar density with Galactic latitude.

In re-running the position comparison routine described in Section $3 \cdot 1$, the appropriate object density and radio-optical separation were used to calculate the chance probability for each potential identification. Note that this calculation is different from Figure 2 as it only takes account of the object density. A reliability limit was imposed by discarding associations for unresolved sources with a chance probability of more than $2 \%$. Extended sources, for which real radio-optical position offsets can occur, were inspected individually as described in the next section.

\section{Inspection of Plates and the Digitized Sky Survey}

Having generated a list of preliminary identifications using COSMOS, we then inspected the actual sky images at each radio position using either the survey films directly or, more conveniently, the Digitized Sky Survey (DSS; White, Postman \& Lattanzi 1993). The DSS is a digital reproduction on CD-ROM of images of the whole sky from blue sensitive Palomar $\mathrm{O}$ and UK Schmidt IIIaJ plates; the pixel size is $1^{\prime \prime} .7$. It should be pointed out that COSMOS and DSS have complementary rôles in the identification process: the COSMOS parameters cannot readily be generated from the DSS, and examination of DSS images is necessary for checking COSMOS classifications.

Visual inspection, usually via radio-DSS overlays, is an essential step in securing identifications for very extended sources. For such sources a simple position comparison is inadequate, because the host galaxy may be displaced significantly from any radio peak position, e.g. a double-lobed source without a detectable core component. When such structures were 


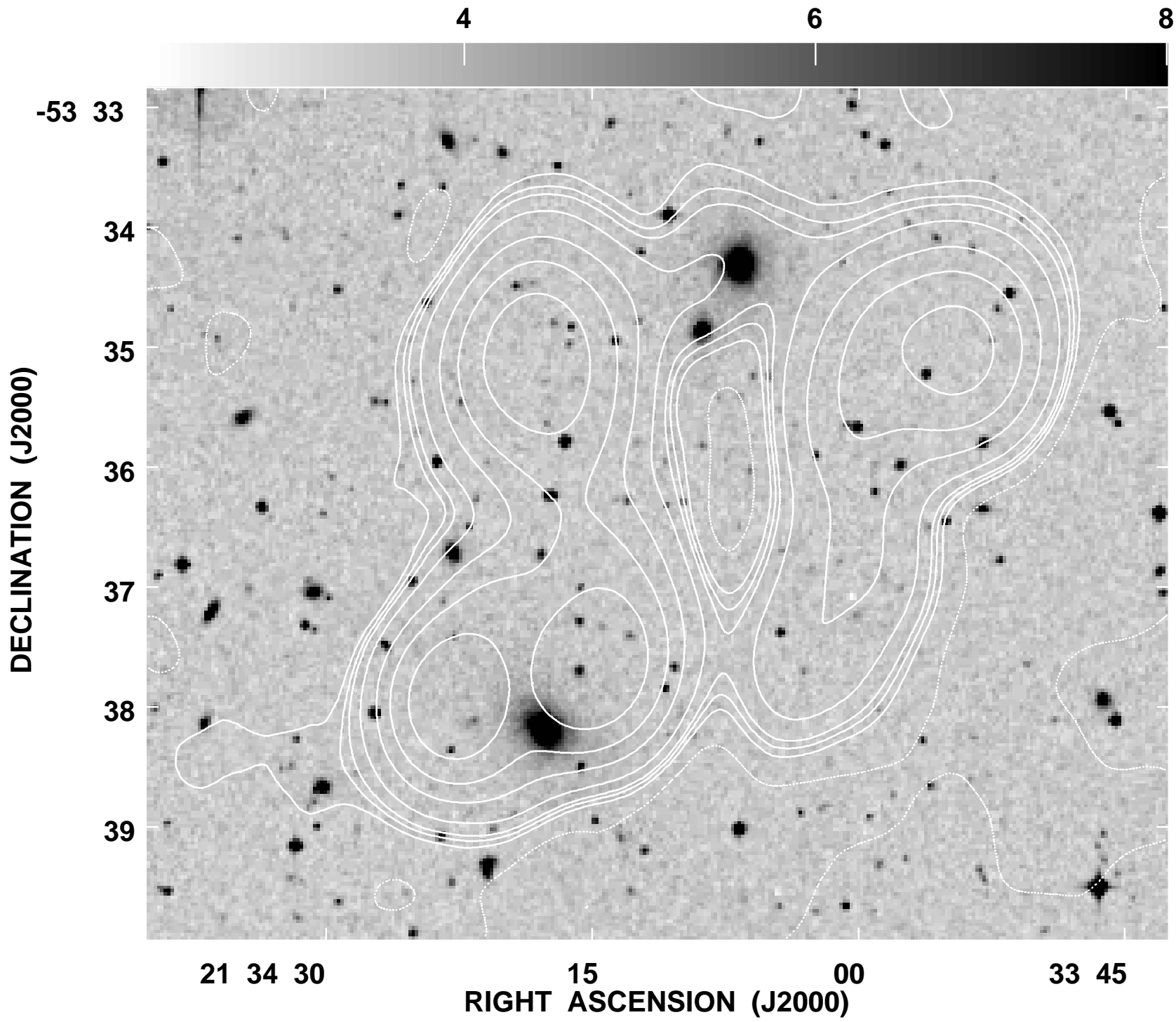

Figure 3-A computer generated overlay of the MOST image (contours) on the Digitized Sky Survey (grey scale) for a complex radio source near the centre of the cluster A3785. The contour levels are $-4,4,7,10,20,50,100,200$ and 600 $\mathrm{mJy} /$ beam. We expect that some or all of the radio emission is associated with the two brightest galaxies, although their relative contributions are difficult to determine at this resolution.

recognised, the separate positions and flux densities were combined and listed as one source. The situation is further complicated in clusters where the morphologies of sources can often be highly distorted by drag forces arising from galaxy motion through the intracluster medium, from buoyancy forces, or from winds in the intra-cluster medium, all leading to significant displacements of the galaxy from the radio centroid.

Overlays of MOST contours on the corresponding optical images from the DSS were made using a series of programs in AIPS. In most cases it was possible to confirm or reject identifications by inspecting hard copies of these overlays, but occasionally it was necessary to inspect the original sky survey material. In a few cases it was not possible to make an identification with confidence, because the radio morphology was unusual or difficult to classify at the resolution of the MOST, and/or there was more than one plausible counterpart. An example is shown in Figure 3; sources such as this have been targeted for higher resolution observations with the Australia Telescope Compact Array (ATCA) to help clarify the identifications.

Experience showed that it was not possible to apply a rigid set of criteria but the following broad guidelines were followed in the inspection process, keeping in mind the results from the Monte Carlo tests described in Section $3 \cdot 2$ and Figure 2.

(1) If a visual inspection confirmed a COSMOS galaxy classification and the source was unresolved or very slightly resolved, the identification was retained unless the $2 \%$ chance probability limit was exceeded.

(2) If a visual inspection confirmed a stellar COSMOS classification, a possible QSO identification was accepted (subject to the $2 \%$ 
chance probability limit) if the automated process claimed it. No spectroscopic information was available for confirmation, and there is currently no colour information in readily accessible digital form.

(3) If the object was classified as 'faint' by COSMOS, and it could be clearly classified by visual inspection (only a small fraction), the above rules were applied. If a clear classification was impossible, the identification was retained if $R \leq 1$ and the $2 \%$ chance probability limit was not exceeded. Such faint objects were kept separate from the galaxy and possible QSO identifications.

(4) If the radio source was well resolved, or showed complex structure, it was assumed to be associated with a galaxy. The search for counterparts took into account what is commonly known about the structures of radio sources and how they can be distorted by the cluster environment. Galaxies between the lobes of double sources were considered, as were galaxies in the elbows of bent structures and at either end of elongated structures. In order to preserve reliability, only relatively bright galaxies $\left(b_{J}<19\right.$, see Figure $\left.2 \mathrm{~d}\right)$ were considered as counterparts to complex extended sources. Extended sources often had strong cores associated with bright galaxies, but this was not always the case.

(5) There were 14 instances where two or more objects satisfied the above identification criteria. In those cases the following course was followed:

- Galaxies were preferred over stellar objects, unless the galaxy offset was many times greater.

- For two objects of the same type the closer one was normally preferred, unless the difference in separation was much less than the separations themselves in which case the brighter one was preferred.

In a few cases, a faint object or even a bright galaxy was not listed in the COSMOS catalogue and positions were measured directly from the DSS. The number of such objects is too small to significantly affect the identification statistics.

\section{Results}

In the 27 images studied, a total of 1030 radio peaks were detected above our $5 \sigma$ cutoff. A small fraction of these were deemed to be multiple, either because they were merged in the image or because two or more peaks were clearly associated with a particular galaxy. The final source count of 927 is approximate because of uncertainty over which sources are genuine doubles and which are unrelated
Table 2. Optical identifications for 927 radio sources in 27 cluster fields

\begin{tabular}{lcc}
\hline Object type & $\begin{array}{c}\text { Number of } \\
\text { identifications }\end{array}$ & $\begin{array}{c}\text { Percentage of } \\
\text { identifications }\end{array}$ \\
\hline Galaxies & 191 & $21 \pm 1 \cdot 5$ \\
Possible QSOs & 43 & $4 \cdot 6 \pm 0 \cdot 7$ \\
\hline
\end{tabular}

point sources with a small projected separation. Table 2 shows the numbers and percentages of galaxies and stellar objects identified. The quoted (Poisson) errors indicate the significance of the observed fraction of identifications as a measure of the underlying average rate. Recall that we imposed a $2 \%$ chance probability limit. Given that about $26 \%$ of our sources have identifications we expect that at most $(2 / 26) \times 100=7 \cdot 7 \%$ are chance coincidences, i.e. the identification list is more than $92 \%$ reliable. The unidentified sources are expected to be associated with objects below the plate limit; results from Windhorst, Kron \& Koo (1984) suggest that the majority of these will be galaxies.

Our results can be compared with those of Unewisse (1993), who searched for optical identifications for 682 MOST radio sources in the fields of nearby rich clusters down to similar flux density limits, and found 173 (25\%) non-stellar and $34(5 \%)$ stellar identifications. We find the same fraction of possible QSO identifications and a slightly smaller fraction of galaxy identifications, but the difference is not significant.

\section{Cluster Membership}

In a first attempt at separating cluster members from interlopers in our sample, we have made use of the data compiled by Auriemma et al. (1977) on the local radio luminosity function of elliptical galaxies. By combining radio data on galaxies from four different published lists, they showed that galaxies having radio emission above detection limits similar to ours span absolute photographic (nearly equivalent to blue) magnitudes from -18.5 to -22.5 for our adopted $H_{0}$. Absolute magnitudes were calculated for all of our identified radio galaxies, assuming they were at the cluster distance. Those falling outside the above range are probably interlopers (mostly background galaxies), while those within this range are likely to be cluster members.

We show in Figure 4 the projected spatial distribution of radio galaxies with magnitudes falling in the above range for the 23 clusters of distance class $\mathrm{D} \leq 4 \quad(z \leq 0 \cdot 072)$; the distance class restriction allows us to consider a complete sample. As in previous studies (e.g. Mills and Hoskins 1977; Robertson \& Roach 1990; Unewisse 1993; Ledlow \& Owen 1995a), we find a large excess of radio sources towards the centres of the clusters, and a low but uniform spread in the outer regions, suggesting 


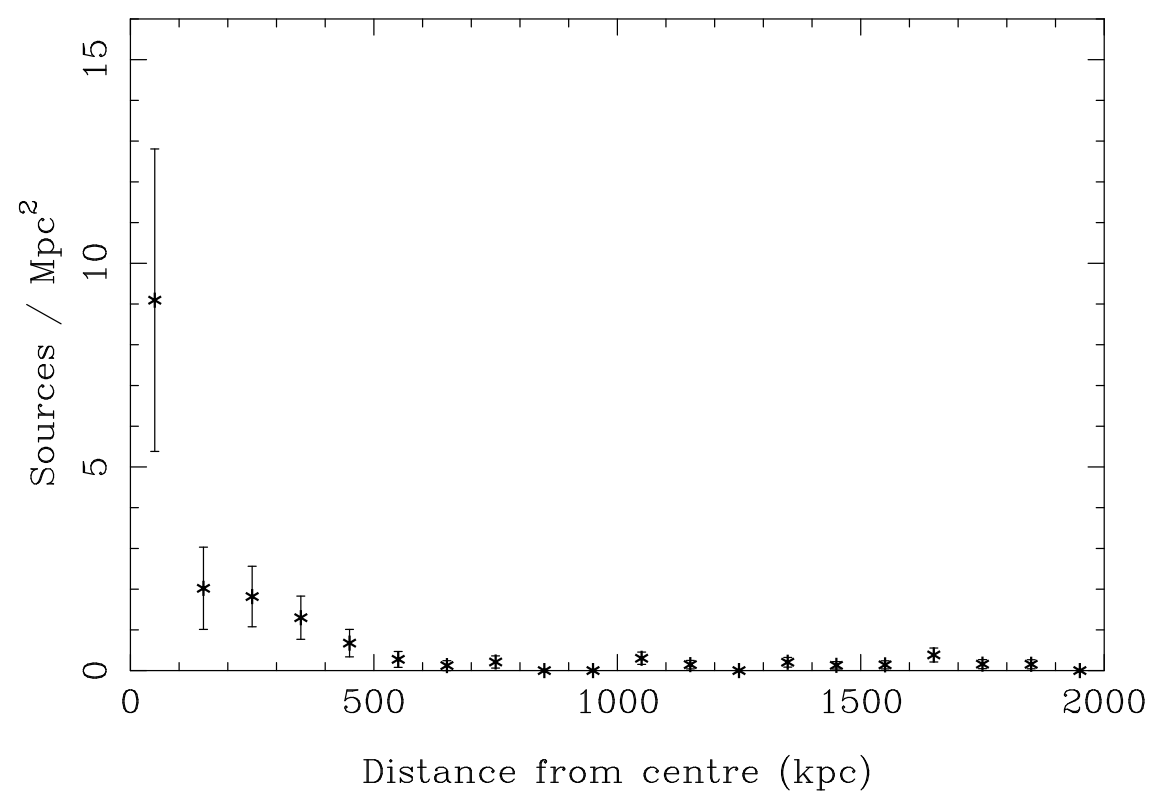

Figure 4-Radial distribution of identified radio galaxies selected from $23 \mathrm{D} \leq 4$ clusters on the basis of their absolute magnitudes (see Section 6). The Abell radius is $R_{A}=2000 \mathrm{kpc}$.

that non-cluster members may be contaminating the distribution. Although refinement of the separation process will be necessary, the background is considerably reduced compared with previous work.

An interesting result from Figure 4 is the excess over the background for projected distances between 100 and $500 \mathrm{kpc}$ from the cluster centre. A similar feature was reported by Robertson \& Roach (1990) but is seen here with greater contrast because of the successful removal of most of the background population, together with a more thorough identification procedure, especially for extended sources. Since the radial averaging in Figure 4 acts to smear out the radio galaxy distribution, our follow-up analysis of this sample will use the COSMOS database to examine more closely the radio detection rate as a function of local galaxy density.

\section{Conclusions}

We have found that the COSMOS/UKST Southern Sky Object Catalogue is a valuable resource for making optical identifications of MOST radio sources. Tentative identifications can be made quickly and efficiently for unresolved and slightly resolved sources, but users need to be aware of occasional object misclassifications. Visual inspection is the only practical way to identify complex extended sources, especially nearby cluster sources with highly distorted morphologies. Overlays of radio images on the DSS provide the most convenient method for assessing the reliability of such associations. In a first attempt to separate cluster radio galaxies from interlopers, we find a concentration strongly peaked towards the centres of the clusters, together with a lesser concentration extending out to $\sim 500 \mathrm{kpc}$.

\section{Acknowledgments}

AJH thanks fellow members of the Astrophysics Department at the University of Sydney for sharing their experience in many useful discussions which assisted in the development of techniques described here, and especially Taisheng Ye for reducing the Molonglo data prior to the commencement of this study and for determining the rms noise values.

We thank the anonymous referee for helpful comments.

This research has used data from the COSMOS/UKST Southern Sky Catalogue provided by the Anglo-Australian Observatory and has made use of the NASA/IPAC Extragalactic Database (NED) which is operated by the Jet Propulsion Laboratory, Caltech, under contract with the US National Aeronautics and Space Administration. The Molonglo Observatory Synthesis Telescope is funded by the Australian Research Council and the University of Sydney. AJH acknowledges the support of a Sydney University Postgraduate Scholarship from the School of Physics.

Abell, G. O., Corwin, H. G., \& Olowin, R. P. 1989, ApJ Supp. Ser., 70, 1

Allington-Smith, J. R., Perryman, M. A. C., Longair, M. S., Gunn, J. E., \& Westphal, J. A. 1982, MNRAS, 201, 331

Andernach, H., Tie, H., Sievers, A., Reuter, H. P., Junkes, N., \& Wielebinski, R. 1988, A\&A Suppl. Ser., 73, 265

Auriemma, C., Perola, G. C., Ekers, R., Fanti, R., Lari, C., Jaffe, W. J., \& Ulrich, M. H. 1977, A\&A, 57, 41

Collins, C. A., Heydon-Dumbleton, N. H., \& MacGillivray, H. T. 1989, in The World of Galaxies, ed. H. Corwin et al. (Berlin: Springer), 21

Condon, J. J. 1996, Errors in elliptical gaussian fits. Electronic preprint: http://www.cv.nrao.edu/jcondon/nvss.html 
Drinkwater, M. J., Barnes, D. G., \& Ellison, S. L. 1995, PASA, 12, 248

Fanti, C., Fanti, R., Feretti, L., Gioia, I. M., Giovannini, G., Gregorini, L., Marano, B., Padrielli, L., Parma, P., Tomasi, P., \& Zitelli, V. 1983, A\&A Suppl. Ser., 52, 411 Large, M. I., Mills, B. Y., Little, A. G., Crawford, D. F., \& Sutton, J. M. 1981, MNRAS, 194, 693

Ledlow, M. J., \& Owen, F. N. 1995a, AJ, 109, 853

Ledlow, M. J., \& Owen, F. N. 1995b, AJ, 110, 1959

Ledlow, M. J., \& Owen, F. N. 1996, AJ, 112, 9

Mills, B. Y., \& Hoskins, D. G. 1977, Aust. J. Phys., 11, 360 Owen, F. N., White, R. A., Hilldrup, K. C., \& Hanish, R. I. 1982, AJ, 87, 1083

Reynolds, J. E. 1986, PhD thesis, University of Sydney

Robertson, J. G. 1991, Aust. J. Phys., 44, 729
Robertson, J. G., \& Roach, G. J. 1990, MNRAS, 247, 387 Slee, O. B., Roy, A. L., \& Savage, A. 1994, Aust. J. Phys., 47,145

Sodré, L., Capelato, H. V., Steiner, J. E., Proust, D., \& Mazure, A. 1992, MNRAS, 259, 233

Unewisse, A. M. 1993, PhD thesis, University of Sydney

Unewisse, A. M., Hunstead, R. W., \& Piestrzynski, B. 1993, PASA, 10, 229

White, R. L., Postman, M., \& Lattanzi, M. G. 1993, in Astronomy for Wide-field Imaging, ed. H. T. MacGillivray et al. (Dordrecht: Kluwer), 167

Windhorst, R. A., Kron, R. G., \& Koo, D. C. 1984, A\&A Suppl. Ser., 58, 39 